\title{
CARACTERIZAÇÃo MORFOLÓGICA E AVALIAÇÃo DE CULTIVARES DE BANANEIRA RESISTENTES A SIGATOKA NEGRA (Mycosphaerella fijiensis Morelet) NO SUDOESTE GOIANO ${ }^{1}$
}

\author{
SILVIA CORREA SANTOS ${ }^{2}$, LUCIANA CELESTE CARNEIRO ${ }^{3}$, AMÉRICO NUNES DA SILVEIRA NETO3, \\ EUTER PANIAGO JÚNIOR ${ }^{3}$, HENRIQUE GONÇALVES DE FREITAS ${ }^{4}$, CECÍLIA NASCIMENTO PEIXOTO
}

\begin{abstract}
RESUMO - Cinco cultivares de bananeira resistentes a Sigatoka Negra foram cultivadas na área experimental da Universidade Federal de Goiás, no município de Jataí, localizado na região Sudoeste do Estado. O objetivo foi introduzir genótipos resistentes a Sigatoka Negra no Estado, bem como fazer a caracterização morfológica desses materiais nessas condições climáticas. O trabalho foi realizado por meio da avaliação, em três ciclos produtivos, dos seguintes componentes de produção: intervalo em dias entre o plantio e o florescimento, número de folhas no florescimento e na colheita, número de pencas por cacho, frutos por cacho e frutos por penca, comprimento do cacho e dos frutos e peso do engaço, das pencas e do cacho. As cultivares falsa FHIA-18 e Caipira produziram em média aos 393 dias, apresentando maior precocidade em relação às demais cultivares. Como era esperado, a FHIA-21, que é uma bananeira tipo Terra, apresentou um ciclo maior (488 dias). O clima e a altitude de Jataí-GO podem ter contribuído para o aumento do ciclo das cinco cultivares quando comparado com outras regiões. Para as características de produção, a FHIA-01 e a falsa FHIA-18 se destacaram das demais. Para o número de pencas, a Thap Maeo se destacou nos três ciclos, mostrando a característica deste genótipo. As maiores alturas na floração foram observadas nas cultivares FHIA21 e Thap Maeo, que também foram as que mais sofreram com os efeitos do vento.
\end{abstract}

Termos para Indexação: Musa spp, genótipos resistentes, componentes de produção

\section{MORPHOLOGIC CHARACTERIZATION AND EVALUATION OF BANANA CULTIVARS RESISTANT TO THE BLACK SIGATOKA DISEASE (Mycosphaerella fijiensis Morelet) IN THE SOUTHWEST OF THE STATE OF GOIÁS, BRAZIL.}

\begin{abstract}
Five banana cultivars resistant to the Black Sigatoka Disease were grown at the experimental field of the Universidade Federal de Goiás (UFG), located in the Southwest region of the State of Goiás (Brazil). This work aimed the introduction of Black Sigatoka resistant cultivars as well as the characterization of the morphological aspects of such cultivars at that climatic region. The following characteristics were evaluated, in the first three cycles: days between planting and flowering, leaf number at flowering and at harvesting, fruit and hand number per bunch and fruit number per hand. A canonical variable analysis was also done, by the Tocher method. The evaluated characteristics were similar during the three growing seasons, insuring the morphological characterization of such genotypes in this climatic region. The cultivars false FHIA 18 and Caipira were harvested 393 days after planting data, showing highest precocity among the other cultivars. As expected, FHIA 21, a plantain type cultivar, showed the highest growing cycle (488 days after planting data). The climatic condition and the elevation of that experimental area may have helped to increase the growing cycle of those cultivars when in comparison to other climatic regions. For the harvesting characteristics, the cultivars FHIA-01 and false FHIA-18 promoted the best results compared to the other ones. For hand number, the cultivar Thap Maeo had the best results during the three growing seasons, establishing that morphological characteristic for that genotype. Plant height at flowering was highest for FHIA-21 and Thap Maeo and both cultivars were mostly damaged by hard winds. Index Terms: Musa spp, cultivars resistance, crop components
\end{abstract}

\section{INTRODUÇÃO}

A banana é cultivada em todas as regiões quentes do mundo, produz durante quase todo o ano, é consumida no mundo inteiro e movimenta a economia de diversos países produtores. É bem apreciada no mundo, por suas características organolépticas, perdendo apenas para a laranja em comercialização. Os países da América Latina são os maiores exportadores de banana, com o domínio de $80 \%$ do mercado, sendo o Equador o maior exportador de banana. Os Estados Unidos são os maiores importadores, com $33 \%$ do mercado de banana, já o Brasil é o maior consumidor e a Índia o maior produtor no mundo (FAO, 2003).

O Brasil é o segundo maior produtor de banana do mundo. Em 2005, a colheita foi de 6,6 milhões de toneladas e movimentou cerca de $\mathrm{R} \$ 2,5$ bilhões. Além do subgrupo Cavendish, as variedades Prata e Maçã também são produzidas e consumidas no Brasil. O
Estado de São Paulo é o principal produtor, com 16,4\% do mercado. Os paulistas colheram 1,1 milhão de toneladas da fruta, ficando em primeiro lugar no ranking dos Estados produtores e em segundo lugar em produtividade média, com cerca de 22.300 quilos por hectare. A Bahia colheu 867.392 toneladas da fruta, seguida de Santa Catarina, com 654.862 toneladas. O melhor desempenho em termos de produtividade ficou com o Rio Grande do Norte, com média de 31.400 quilos por hectare, embora a safra tenha sido de $200 \mathrm{mil}$ toneladas. As vendas externas de banana, no ano de 2005, renderam ao Brasil cerca de US\$ 27 milhões, e os países da União Européia foram os principais compradores (ROSA et al., 2006).

No Estado de Goiás, a cultura da banana destaca-se como uma das principais fruteiras cultivadas, com grande importância social. Segundo dados da SEAGRO (2006), foram colhidos no Estado cerca de 13,2 mil hectares de banana no ano de 2005, que apresentou um rendimento médio em torno de 11,5 t.ha $^{-1}$. Os principais

\footnotetext{
${ }^{1}$ (Trabalho 205-2005). Recebido: 16-12-2005. Aceito para publicação: 17-11-2006.

${ }^{2}$ Eng. Agrônoma, Dra., Professor Adjunto, Coordenadora do projeto/Universidade Federal de Goiás/Campus de Jataí/CCAB- Rod. BR 364, Km192, n.1800, Setor Parque Industrial. Jataí-GO. CEP: 75800-000. E-mail: scscorrea@yahoo.com.br.

${ }^{3}$ Professores de Campus de Jataí, E-mails: celeste@esalq.usp.br; americonunesagro@yahoo.com.br; euter@abenet.com;

${ }^{4}$ Aluno do Curso de Agronomia, Bolsista. E-mail: rico21agro@ yahoo.com.br;

${ }^{5}$ Superintendência Federal de Agricultura de Goiás. Praça Cívica n. 100, Centro, Goiânia-GO, CEP: 74003-010, E-mail: ceciliapeixoto3@ hotmail.com
} 
municípios produtores são: Anápolis, Itaguaru, Anicuns, Buriti Alegre, Jataí, Pirenópolis e Santa Fé de Goiás, sendo Goiás o segundo colocado regional, tanto em área plantada como em volume de produção.

Apesar de existir um grande número de variedades de banana no Brasil, considerando a preferência dos consumidores, produtividade, tolerância às doenças, altura de planta e resistência à seca e ao frio, poucas apresentam potencial agronômico que podem ser indicadas para fins comerciais. As mais difundidas são cultivares do subgrupo Cavendish, como Nanica, Nanicão e Grande Naine, e cultivares do grupo AAB, como Prata, Prata-Anã e Pacovan, do subgrupo Prata; Terra e D'Angola, do subgrupo Terra; e Maçã e Mysore. Entretanto, todas apresentam pelo menos uma característica indesejável, como altura de planta inadequada ou suscetibilidade a alguma doença (SILVA et al., 1999). Trabalhos que envolvam avaliações de cultivares nas diferentes regiões são importantes e oferecem aos produtores opções de cultivo, além de colaborar com o desenvolvimento regional da cultura (SILVA et al., 2006; DONATO et al., 2006; PEREIRA et al., 2004; MOURA et al., 2002; ANDRADE et al.,2002; SILVA et al., 2000 ).

A Sigatoka Negra, doença causada pelo fungo Mycosphaerella fijiensis Morelet, é o principal problema fitossanitário da banana na América Central, México, Panamá, Colômbia, Equador e países da África e Ásia. Causa destruição rápida das folhas, afetando o crescimento e a produtividade das plantas, devido ao comprometimento da capacidade fotossintética. As plantas doentes têm a sua produtividade reduzida e a qualidade da fruta é afetada em razão da maturação precoce. Nos países tradicionalmente produtores de banana, a Sigatoka Negra modificou drasticamente o sistema de produção e as estratégias de controle, aumentando o número de pulverizações anuais, tendo os bananicultores de recorrer à utilização de novos princípios ativos e misturas de fungicidas, resultando em incremento no custo de produção (HINZ, 2000).

A doença foi identificada no Brasil em 1998, primeiramente no Estado de Amazonas e disseminou-se para o resto do País, mas há Estados, como Goiás (instrução normativa 29 de 08-06-2006), Minas Gerais, Pernambuco e Rio de Janeiro, considerados pelo Ministério da Agricultura livres da Sigatoka Negra (SEAGRO, 2006).

Embora ainda não se tenha constatado a sua presença em Goiás, a Sigatoka Negra é uma preocupação Estadual, em virtude principalmente de sua localização geográfica com Estados onde a doença já se estabeleceu. Esta localização coloca-o em uma área de risco, principalmente nas divisas e nas rodovias que constituem a rota de produtos agrícolas provenientes de regiões onde a doença já ocorre. Esse fato sinaliza que seu ingresso no sudoeste Goiano, onde está inserido o município de Jataí, pode ser apenas uma questão de tempo.

Apesar de seu maior dano em relação à Sigatoka Amarela, grande parte das recomendações de controle disponíveis acerca dessa doença é aplicável a Sigatoka Negra, inclusive em relação aos produtos utilizados no controle químico. Mas, apesar de a Sigatoka Amarela estar presente de forma generalizada e causar perdas e prejuízos aos bananicultores goianos, no município de Jataí, não é comum o controle químico desta doença, sendo necessário o uso de cultivares resistentes para que, caso a Sigatoka Negra venha a se estabelecer, seja possível e viável a sua convivência.

Entretanto, sabe-se que as dificuldades atualmente encontradas no controle da Sigatoka Negra são devidas, principalmente, à queda na eficiência dos produtos químicos. Neste sentido, o melhoramento genético de bananeiras, visando a variedades resistentes, é uma linha de pesquisa prioritária, devido à crescente dispersão da Sigatoka Negra no mundo e ao impacto econômico que ela representa (Orozco-Santos, 1998).

Sendo assim, o objetivo deste trabalho foi introduzir e caracterizar cultivares de bananeira resistentes a Sigatoka Negra nas condições edafoclimáticas de Jataí, no sudoeste Goiano, região tipicamente produtora de banana do subgrupo Terra, bem como obter informações sobre o comportamento produtivo destes genótipos na região.

\section{MATERIAL E MÉTODOS}

O experimento foi conduzido em área experimental no Centro de Ciências Agrárias do Câmpus Avançado de Jataí, da Universidade Federal de Goiás, $17^{0} 53^{\circ}$ 'S e $52^{\circ} 43^{\circ} \mathrm{W}$, e 670m de altitude, localizada no município de Jataí. O município está situado na microrregião do sudoeste Goiano, com temperatura média anual de $22^{\circ} \mathrm{C}$, e uma precipitação média anual variando de 1.650 a $1.800 \mathrm{~mm}$. O solo da área do experimento é um Latossolo Roxo distrófico, textura arenosa (Mariano \& Scopel, 2001). Os dados de precipitação e temperatura registrados durante os três ciclos produtivos são apresentados nas Tabelas 1 e 2 .

O ensaio foi instalado em dezembro de 2001, em delineamento experimental em Blocos Casualizados, com seis tratamentos (cultivares): Caipira, Thap Maeo, FHIA-01, FHIA-21, falsa FHIA-18 e Red Yade (subgrupo Terra, não resistente a Sigatoka Negra), fornecidas pela Delegacia Federal de Agricultura do Estado de Goiás, oriundas da Empresa Campo Biotecnologia (Paracatu- MG), com exceção da Red Yade, que foi propagada por rizoma. O bananal recebeu todos os tratos culturais recomendados para a cultura, irrigação por microaspersão durante os ciclos produtivo e um programa de adubação conforme Ribeiro et al. (1999). Foram avaliados os seguintes componentes de produção: a) Intervalo em dias entre o plantio e o florescimento - avaliado por meio de inspeções semanais no bananal para a determinação da data de florescimento de cada planta, para a posterior determinação da precocidade das cultivares; b) Número de folhas no florescimento e na colheita avaliado por meio da contagem de folhas vivas, por ocasião da floração e da colheita; c) Número de pencas por cacho, frutos por cacho e frutos por penca - Os cachos foram colhidos no estádio $3 / 4$ normal, com os frutos apresentando quinas e os lados mais largos, iniciando a fase arredondada com cerca de $32-34 \mathrm{~mm}$ de diâmetro. Após a colheita, os cachos foram despencados, para a contagem do número de pencas e o número de frutos; d) Comprimento do cacho e dos frutos - Após o despencamento, foi determinado o tamanho dos cachos, medindo-se, no engaço, a distância entre as inserções da primeira e última pencas. Para determinar o tamanho dos frutos, mediu-se a parte central e interior de cada penca (parte voltada para o engaço). Além disto, foi medido o peso e tamanho (diâmetro e comprimento) dos frutos da 2 a penca; e) Peso do engaço, das pencas e do cacho - O engaço foi pesado, delimitando o seu tamanho a 15 $\mathrm{cm}$ abaixo da inserção da última penca, determinada quando do corte do coração, e a $15 \mathrm{~cm}$ acima da inserção da primeira penca. As pencas foram pesadas individualmente, após contagem e medição dos frutos.

\section{RESULTADOS E DISCUSSÃO}

Foram avaliados por três ciclos consecutivos o comportamento produtivo das seis cultivares nas condições bioclimáticas da região de Jataí-GO (Tabelas 3; 4 e 5).

Em relação ao primeiro ciclo, a cultivar falsa FHIA-18 floresceu aos 244 dias após o plantio, sendo a mais precoce, seguida das cultivares Caipira (274 dias), Thap Maeo (329 dias), FHIA-01 (340 dias), FHIA-21 e Red Yade (368 dias). Quanto ao período compreendido entre o florescimento e a colheita, a cultivar falsa FHIA-18 completou a maturação aos 150 dias, seguida das cultivares FHIA-21 e Red Yade (120 dias), Caipira (119 dias), FHIA-01 (102 dias) e Thap Maeo (96 dias), assemelhando-se aos valores observados por Andrade et al. (2002) no Piauí. No que tange ao período do plantio à colheita, as cultivares falsa FHIA-18 e Caipira 
TABELA 1 - Dados médios de precipitação e temperatura da Estação Meteorológica - Centro de Ciências Agrárias e Biológicas/INMET-GO $(2002-2003)$

\begin{tabular}{|c|c|c|c|c|c|c|c|c|}
\hline \multirow{3}{*}{ MESES } & \multicolumn{8}{|c|}{ ANOS } \\
\hline & \multicolumn{4}{|c|}{2002} & \multicolumn{4}{|c|}{2003} \\
\hline & $\begin{array}{r}\mathbf{T} \text { máx. } \\
\left({ }^{\circ} \mathrm{C}\right)\end{array}$ & $\begin{array}{r}\text { T mín. } \\
\left({ }^{\circ} \mathrm{C}\right) \\
\end{array}$ & $\begin{array}{r}\text { T méd. } \\
\left({ }^{\circ} \mathrm{C}\right)\end{array}$ & $\begin{array}{r}\text { Precip. } \\
(\mathrm{mm}) \\
\end{array}$ & $\begin{array}{r}\mathbf{T} \text { máx. } \\
\left({ }^{\circ} \mathrm{C}\right) \\
\end{array}$ & $\begin{array}{r}T \text { mín. } \\
\left({ }^{\circ} \mathrm{C}\right)\end{array}$ & $\begin{array}{r}\text { T méd. } \\
\left({ }^{\circ} \mathrm{C}\right)\end{array}$ & $\begin{array}{r}\text { Precip. } \\
(\mathbf{m m})\end{array}$ \\
\hline Jan & 33,7 & 16,2 & 24,0 & 323,2 & 31,7 & 19,2 & 24,3 & 239,7 \\
\hline Fev & 32,3 & 18,3 & 23,0 & 416,7 & 34,9 & 17,6 & 24,2 & 229,1 \\
\hline Mar & 33,3 & 17,0 & 24,1 & 318,8 & 34,7 & 18,2 & 23,5 & 267,7 \\
\hline Abr & 33,3 & 13,8 & 23,6 & 29,1 & 33,2 & 11,6 & 22,6 & 128,0 \\
\hline Maio & 32,9 & 8,5 & 21,4 & 62,8 & 32,2 & 4,5 & 19,0 & 32,1 \\
\hline Jun & 31,8 & 9,2 & 19,5 & 0,0 & 31,5 & 6,9 & 19,2 & 0,0 \\
\hline Jul & 33,6 & 6,7 & 19,9 & 28,3 & 33,1 & 6,9 & 19,0 & 0,0 \\
\hline Ago & 34,7 & 11,6 & 22,8 & 19,2 & 34,8 & 3,5 & 20,2 & 10,1 \\
\hline Set & 35,7 & 2,8 & 22,4 & 38,6 & 39,0 & 10,7 & 24,0 & 21,6 \\
\hline Out & 37,2 & 18,0 & 25,4 & 69,5 & 36,4 & 14,8 & 24,5 & 195,0 \\
\hline Nov & 35,8 & 15,3 & 25,0 & 95,8 & 36,0 & 16,0 & 24,4 & 58,0 \\
\hline Dez & 35,5 & 18,8 & 24,9 & 267,5 & 34,4 & 16,7 & 24,6 & 303,9 \\
\hline $\begin{array}{l}\text { Média } \\
\text { Total }\end{array}$ & 34,2 & 13,0 & 23,0 & 1669,5 & 34,3 & 12,2 & 22,5 & 1485,2 \\
\hline
\end{tabular}

TABELA 2 - Dados médios de precipitação e temperatura da Estação Meteorológica - Centro de Ciências Agrárias e Biológicas/INMET$\mathrm{GO}(2004)$.

\begin{tabular}{lcccc}
\hline \multirow{2}{*}{ MESES } & \multicolumn{4}{c}{ ANO - 2004 } \\
\cline { 2 - 5 } & T máx. $^{\left({ }^{\circ} \mathbf{C}\right)}$ & T mín. $\left.^{\circ}{ }^{\circ} \mathbf{C}\right)$ & T méd. $\left({ }^{\circ} \mathbf{C}\right)$ & Precip. $(\mathbf{m m})$ \\
\hline Jan & 30,0 & 20,8 & 23,5 & 267,5 \\
Fev & 29,7 & 20,0 & 23,7 & 363,4 \\
Mar & 31,9 & 17,1 & 24,0 & 125,6 \\
Abr & 30,7 & 18,9 & 23,5 & 124,7 \\
Maio & 27,4 & 15,2 & 20,1 & 37,0 \\
Jun & 26,8 & 11,9 & 19,0 & 15,1 \\
Jul & 26,2 & 11,3 & 18,3 & 8,0 \\
\hline
\end{tabular}

tiveram um ciclo de 393 dias, caracterizando-as como mais precoces. Andrade et al. (2002) também observaram maior precocidade para estas cultivares. A cultivar FHIA-21 apresentou ciclo de 488 dias, superior às demais, como esperado nas cultivares do tipo Terra.

O ciclo apresenta fundamental importância no melhoramento genético da bananeira, pois é um caráter que mostra a expressão da precocidade. A redução do número de dias necessários para a emissão do cacho traz expectativas de retorno do investimento inicial.

A duração do ciclo nas cultivares estudadas diferiu do trabalho realizado por Silva et al. (2000), em Cruz das Almas-BA. Esta apresenta altitude média de $220 \mathrm{~m}$ e temperaturas médias anuais superiores, o que pode ter contribuído para a maior precocidade. A região de Jataí-GO, tem características climáticas diferentes, isto é, as temperaturas médias diminuem a partir de abril e voltam a subir em outubro. Além disto, a altitude local pode ter contribuído no aumento do ciclo.

Nas Tabelas 3 e 4, estão apresentados os componentes de produção das seis cultivares no primeiro e segundo ciclos de produção. Houve incremento em produtividade baseado no caráter peso do cacho do primeiro para o segundo ciclo, exceto para as cultivares FHIA-21 e Red Yade, que tiveram redução de peso de cacho no segundo ciclo. Na cultivar falsa FHIA-18, observou-se o maior incremento (55\%). No primeiro ciclo, as cultivares FHIA-01, FHIA-21 e Red Yade foram superiores em peso de cacho. Os cachos da Red Yade tiveram de ser tutorados até completar o ciclo. No 2o ciclo, destacaram-se em peso de cacho a FHIA-01 e a falsa FHIA-
18. No trabalho desenvolvido por Silva et al. (2000), apenas o híbrido FHIA-01, no primeiro ciclo, apresentou desempenho inferior, enquanto as demais cultivares exibiram rendimentos superiores. $\mathrm{O}$ primeiro ciclo não é o momento oportuno para analisar o peso do cacho na maioria dos genótipos, uma vez que o caráter pode aumentar do primeiro para o segundo ciclo da cultura (SILVA et al., 2002)

Quanto à característica número de pencas, a Thap Maeo destacou-se das demais no primeiro ciclo, igualando-se a FHIA-01 e à falsa FHIA-18 no segundo ciclo. A Thap Maeo apresentou maturação desuniforme, isto é, alguns "dedos" da penca amadureciam ainda no campo, antecedendo o período de colheita, A Caipira e a FHIA-21 apresentaram, em média, três pencas a menos que a Thap Maeo. Moura et al. (2002) observaram, para as cultivares FHIA-01 e falsa FHIA-18, valores médios de 7,1 e 5,7 pencas/cacho, respectivamente. $\mathrm{O}$ número de pencas e o número de frutos são importantes no melhoramento genético da bananeira, pois influenciam diretamente no tamanho e no peso do cacho, que expressam a produtividade de um genótipo (SILVA et al., 2000).

O trabalho de Pereira et al. (2004) relata que a Thap Maeo $(\mathrm{AAB})$ apresenta alta resistência ao despencamento, enquanto a falsa FHIA-18 e a FHIA-01 tiveram valores intermediários ao despencamento. Nas condições de Jataí-GO, observou-se maior queda natural para as cultivares falsa FHIA-18 e FHIA-01.

Os descritores peso do cacho e das pencas, embora expressem diretamente a produtividade, não podem ser considerados 
TABELA 3 - Caracterização do desenvolvimento e do rendimento das cultivares de bananeira, avaliadas no primeiro ciclo, UFG/Jataí-GO.

\begin{tabular}{|c|c|c|c|c|c|c|c|c|c|c|c|c|c|}
\hline \multirow[t]{2}{*}{ Tratamentos } & \multicolumn{13}{|c|}{ Características $^{2}$} \\
\hline & $\overline{\mathbf{P C}^{1}}$ & $\mathbf{N P}$ & $\mathbf{C E}$ & $\overline{\mathbf{D E}}$ & $\mathbf{P E}$ & $\varnothing \mathbf{2}^{\mathbf{a}} \mathbf{P}$ & $\mathbf{C 2}{ }^{\mathrm{a}} \mathbf{P}$ & $\mathbf{P 2}{ }^{\mathbf{a}} \mathbf{P}$ & $\mathbf{N} 2^{\mathrm{a}} \mathbf{P}$ & $\varnothing$ PSFL & $\begin{array}{l}\text { NFF } \\
\end{array}$ & $\overline{\text { NFC }}$ & APF \\
\hline FHIA-01 & $14,95 \mathbf{a}^{1}$ & $7,77 \mathbf{b}$ & $52,76 \mathbf{a b}$ & $57,94 \mathbf{a}$ & $1,15 \mathbf{a}$ & $40,78 \mathbf{a}$ & $15,95 \mathbf{b}$ & $2,03 \mathbf{b}$ & $13,51 \mathrm{c}$ & $19,80 \mathbf{a}$ & $13,68 \mathbf{a b}$ & $9,32 \mathbf{a}$ & $2,08 \mathbf{b c}$ \\
\hline FHIA-18 ${ }^{* 2}$ & $8,39 \mathbf{b}$ & $7,28 \mathbf{b c}$ & $31,61 \mathrm{~d}$ & $51,13 \mathbf{b c}$ & $0,43 \mathbf{c}$ & $36,38 \mathbf{b}$ & $11,77 \mathbf{c}$ & $1,14 \mathbf{c}$ & $12,73 \mathbf{c}$ & $18,52 \mathbf{a b}$ & $12,70 \mathbf{b c}$ & $8,70 \mathbf{a b}$ & $1,97 \mathbf{c d}$ \\
\hline FHIA-21 & $15,17 \mathbf{a}$ & $6,23 \mathbf{d}$ & $59,84 \mathbf{a}$ & $55,18 \mathbf{a b}$ & $1,16 \mathbf{a}$ & $42,75 \mathbf{a}$ & $19,84 \mathbf{a}$ & $2,47 \mathbf{a}$ & $12,58 \mathbf{c}$ & $18,99 \mathbf{a b}$ & $12,29 \mathbf{c}$ & $7,49 \mathbf{b}$ & $2,62 \mathbf{a}$ \\
\hline Caipira & $5,07 \mathrm{c}$ & $6,08 \mathbf{d}$ & $23,19 \mathbf{e}$ & $48,84 \mathrm{c}$ & $0,36 \mathbf{c}$ & $29,46 \mathbf{d}$ & $9,08 \mathbf{d}$ & $0,83 \mathbf{c}$ & $17,95 \mathbf{a}$ & $14,30 \mathbf{c}$ & $11,58 \mathbf{c}$ & $8,13 \mathbf{a b}$ & $1,91 \mathbf{d}$ \\
\hline Thap Maeo & $9,76 \mathbf{b}$ & $9,67 \mathbf{a}$ & $46,78 \mathbf{b c}$ & $49,95 \mathbf{c}$ & $0,75 \mathbf{b}$ & $32,97 \mathbf{c}$ & $10,09 \mathrm{~cd}$ & $1,02 \mathrm{c}$ & $15,06 \mathbf{b}$ & $17,88 \mathbf{b}$ & $12,71 \mathbf{b c}$ & $9,49 \mathbf{a}$ & $2,56 \mathbf{a}$ \\
\hline Red Yade & $16,15 \mathbf{a}$ & $6,83 \mathbf{c d}$ & $40,08 \mathbf{c}$ & $59,19 \mathbf{a}$ & $0,85 \mathbf{b}$ & $43,08 \mathbf{a}$ & $19,09 \mathbf{a}$ & $2,59 \mathbf{a}$ & $13,63 \mathbf{c}$ & $19,61 \mathbf{a}$ & $14,34 \mathbf{a}$ & $9,10 \mathbf{a b}$ & $2,16 \mathbf{b}$ \\
\hline
\end{tabular}

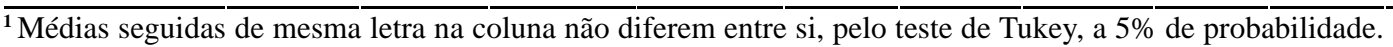

*2 Material introduzido no Brasil como FHIA-18, mas que se trata de material desconhecido.

TABELA 4 - Caracterização do desenvolvimento e do rendimento das cultivares de bananeira, avaliadas no segundo ciclo, UFG/Jataí-GO.

\begin{tabular}{|c|c|c|c|c|c|c|c|c|c|c|}
\hline \multirow[t]{2}{*}{ Tratamentos } & \multicolumn{10}{|c|}{ Características $^{2}$} \\
\hline & $\mathbf{P C ^ { 1 }}$ & $\mathbf{N P}$ & $\mathbf{C E}$ & $\overline{\mathbf{D E}}$ & $\mathbf{P E}$ & $\varnothing \mathbf{2}^{\mathrm{a}} \mathbf{P}$ & $\mathbf{C 2}^{\mathrm{a}} \mathbf{P}$ & $\mathbf{P 2}{ }^{\mathbf{a}} \mathbf{P}$ & $\mathbf{N 2} 2^{\mathrm{a}} \mathbf{P}$ & NFC \\
\hline FHIA-01 & $20,86 \mathbf{a}$ & $9,34 \mathbf{a}$ & $61,82 \mathbf{a}$ & $64,44 \mathbf{a}$ & $1,25 \mathbf{a b}$ & $41,25 \mathbf{a}$ & $77,41 \mathbf{b}$ & $2,38 \mathbf{a}$ & $16,30 \mathbf{c d}$ & $6,24 \mathbf{a}$ \\
\hline FHIA-18 ${ }^{* 3}$ & $18,41 \mathbf{a}$ & $9,56 \mathbf{a}$ & $54,91 \mathbf{a}$ & $69,11 \mathbf{a}$ & $1,62 \mathbf{a}$ & $38,67 \mathbf{a b}$ & $68,95 \mathrm{c}$ & $2,19 \mathbf{a b}$ & $17,46 \mathbf{b c}$ & $5,82 \mathbf{a b}$ \\
\hline FHIA-21 & $11,43 \mathbf{b}$ & $6,10 \mathrm{c}$ & $64,10 \mathbf{a}$ & $51,80 \mathrm{bc}$ & $1,09 \mathbf{b c}$ & $38,74 \mathbf{a b}$ & $82,26 \mathbf{a b}$ & $1,68 \mathrm{bc}$ & $12,76 \mathbf{c d}$ & $4,00 \mathrm{~b}$ \\
\hline Caipira & $9,80 \mathbf{b}$ & $7,39 \mathbf{b}$ & $38,69 \mathbf{b}$ & $53,61 \mathbf{b c}$ & $0,71 \mathrm{c}$ & $31,75 \mathrm{c}$ & $50,86 \mathbf{d}$ & $1,42 \mathrm{c}$ & $21,49 \mathbf{a b}$ & $4,29 \mathbf{a b}$ \\
\hline Thap Maeo & $12,94 \mathrm{~b}$ & $9,60 \mathbf{a}$ & $57,70 \mathbf{a}$ & $55,85 \mathbf{b}$ & $1,01 \mathbf{b c}$ & $33,29 c$ & $54,60 \mathrm{~d}$ & $1,63 \mathbf{b c}$ & $22,60 \mathbf{a}$ & $5,45 \mathbf{a b}$ \\
\hline Red Yade & $10,50 \mathbf{b}$ & $6,10 \mathrm{c}$ & $41,80 \mathbf{b}$ & $48,10 \mathrm{c}$ & $0,68 c$ & $38,43 \mathbf{b}$ & $87,10 \mathbf{a}$ & $1,81 \mathbf{a b c}$ & $12,35 \mathbf{d}$ & $5,93 \mathbf{a b}$ \\
\hline
\end{tabular}

${ }^{1}$ Médias seguidas de mesma letra na coluna não diferem entre si, pelo teste de Tukey, a $5 \%$ de probabilidade.

2 PC: peso do cacho $(\mathrm{kg})$; NP: número de pencas; CE: comprimento do engaço $(\mathrm{cm})$; DE: diâmetro do engaço (mm); PE: peso do engaço (kg);

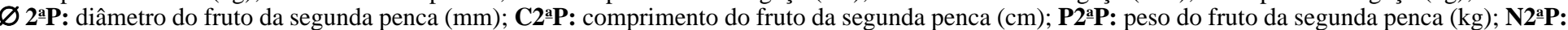
número de frutos da segunda penca; ØPSFL: diâmetro do pseudocaule na floração (cm); NFF: número de folhas na floração; NFC: número de folhas na colheita; APF: altura da planta na floração.

${ }^{* 3}$ Material introduzido no Brasil como FHIA-18, mas que se trata de material desconhecido.

TABELA 5 - Caracterização do desenvolvimento e do rendimento das cultivares de bananeira, avaliadas no terceiro ciclo, UFG/Jataí-GO.

\begin{tabular}{|c|c|c|c|c|c|c|c|c|c|c|}
\hline \multirow[t]{2}{*}{ Tratamentos } & \multicolumn{10}{|c|}{ Características $^{2}$} \\
\hline & $\overline{P C^{1}}$ & $\mathbf{N P}$ & $\mathbf{C E}$ & $\overline{\mathbf{D E}}$ & $\overline{P E}$ & $\varnothing \mathbf{2}^{\mathbf{a}} \mathbf{P}$ & $\mathbf{C 2}^{\mathbf{a}} \mathbf{P}$ & $\mathbf{P 2}^{\mathbf{a}} \mathbf{P}$ & $\mathbf{N} 2^{\mathbf{a}} \mathbf{P}$ & NFC \\
\hline $\begin{array}{l}\text { FHIA-01 } \\
\text { FIA }\end{array}$ & $19,56 \mathbf{a}$ & $10,00 \mathrm{~b}$ & $68,54 \mathbf{a}$ & $64,18 \mathbf{a}$ & $1,74 \mathbf{a}$ & $40,35 \mathbf{a}$ & $83,11 \mathbf{b}$ & $2,51 \mathbf{a}$ & 16,29 bc & $6,21 \mathbf{a}$ \\
\hline FHIA-18 ${ }^{* 3}$ & $17,39 \mathbf{a}$ & $9,80 \mathbf{b}$ & $61,22 \mathbf{a b}$ & $64,48 \mathbf{a}$ & $1,53 \mathbf{a}$ & $38,22 \mathbf{a}$ & $73,65 \mathrm{c}$ & $2,09 \mathbf{a b}$ & 15,17 cd & $5,76 \mathbf{a b}$ \\
\hline FHIA-21 & $11,28 \mathrm{bc}$ & $6,60 \mathrm{~d}$ & $65,32 \mathbf{a b}$ & 48,59 bc & $0,97 \mathbf{b}$ & $40,52 \mathbf{a}$ & $93,03 \mathbf{a}$ & $2,03 \mathrm{~b}$ & 11,49 de & $5,74 \mathbf{a b}$ \\
\hline Caipira & $9,70 \mathbf{c d}$ & $8,20 \mathbf{c}$ & $45,91 \mathbf{c}$ & $53,05 \mathbf{b}$ & $0,91 \mathbf{b c}$ & $32,16 \mathbf{b}$ & $52,14 \mathbf{d}$ & $1,40 \mathrm{c}$ & $20,49 \mathbf{a}$ & $6,08 \mathbf{a}$ \\
\hline Thap Maeo & $12,84 \mathbf{b}$ & $12,20 \mathbf{a}$ & $57,80 \mathbf{b}$ & $48,68 \mathbf{b c}$ & $0,83 \mathbf{b c}$ & $33,50 \mathbf{b}$ & $54,37 \mathbf{d}$ & $1,47 \mathrm{c}$ & $20,20 \mathbf{a b}$ & $5,35 \mathbf{a b}$ \\
\hline Red Yade & $7,64 \mathbf{d}$ & $5,60 \mathbf{e}$ & $42,48 \mathbf{c}$ & $47,09 \mathrm{c}$ & $0,56 \mathbf{c}$ & $39,86 \mathbf{a}$ & 83,48 b & $1,55 \mathrm{c}$ & $9,72 \mathbf{e}$ & $4,90 \mathbf{b}$ \\
\hline
\end{tabular}

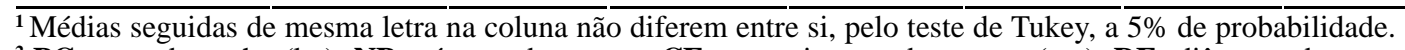

2 PC: peso do cacho (kg); NP: número de pencas; CE: comprimento do engaço $(\mathrm{cm}) ; \mathbf{D E}$ : diâmetro do engaço (mm); PE: peso do engaço (kg); $\varnothing$ 29. P: diâmetro do fruto da segunda penca (mm); C2aP: comprimento do fruto da segunda penca (cm); P2 2 P: peso do fruto da segunda penca (kg); N2aP: número de frutos da segunda penca; ØPSFL: diâmetro do pseudocaule na floração $(\mathrm{cm})$; NFF: número de folhas na floração; NFC: número de folhas na colheita; APF: altura da planta na floração.

${ }^{* 3}$ Material introduzido no Brasil como FHIA-18, mas que se trata de material desconhecido.

isoladamente na escolha de uma cultivar, pois outros caracteres relacionados aos frutos, como peso, comprimento, diâmetro, sabor e resistência ao despencamento, devem ser considerados.

Nas avaliações feitas na segunda penca, observou-se que as médias do diâmetro do fruto da segunda penca foram maiores para as cultivares FHIA-01 e FHIA-21 no primeiro ciclo. Já no segundo ciclo, destacaram-se a FHIA-01, falsa FHIA-18 e FHIA-21. Para o comprimento do fruto da 2 2 penca, destacaram-se as cultivares FHIA-21 e Red Yade com as maiores médias. Os descritores peso do cacho e peso das pencas apresentam estreita relação. No peso do fruto da 2 a penca, as cultivares FHIA-21 e Red Yade se destacaram no primeiro ciclo, mas, no segundo, as cultivares FHIA-01, falsa FHIA-18 e Red Yade apresentaram as maiores médias. Na característica número de frutos da $2^{\underline{a}}$ penca, a cultivar Caipira apresentou a maior média. Já no segundo ciclo, a Caipira e a Thap Maeo apresentaram as maiores médias, diferenciando-se das demais. Estas tiveram boa aceitação no mercado local, principalmente pelo aspecto das pencas, com "dedos" pequenos e atrativos ao consumidor.

Nas características relacionadas ao engaço (peso, comprimento e diâmetro), as cultivares FHIA-01 e FHIA-21 apresentaram as maiores médias no primeiro ciclo. No segundo ciclo de produção, os caracteres relacionados ao engaço mostraram que a FHIA-01, falsa FHIA-18 e FHIA-21 apresentam médias superiores. No trabalho de Moura et al. (2002), em Itambé-PE, as características do engaço se assemelharam com os valores médios observados em Jataí-GO.

Quanto ao diâmetro do pseudocaule na floração, a cultivar Caipira apresentou a menor média, prevalecendo nos ciclos seguintes. A Caipira também apresentou alta capacidade de perfilhamento. Para esta cultivar, durante a condução do bananal, deve-se ter um controle maior no desbaste, a fim de manter a condução e um arranjo adequado. Para o número de folhas na floração, a FHIA-01, falsa FHIA-18 e Thap Maeo apresentaram as maiores médias, refletindo num bom desenvolvimento dos cachos. $\mathrm{Na}$ colheita, não houve diferença entre FHIA-01, falsa FHIA-18, 
Caipira e Thap Maeo quanto ao número de folhas. No segundo ciclo, não houve diferença entre FHIA-01, falsa FHIA-18, Caipira, Thap Maeo e Red Yade em relação ao número de folhas, e estes foram bem inferiores quando comparados ao primeiro ciclo. A perda de folhas pode ter sido influenciada pela ação do vento na área do experimento, que é característico da região. Para os dados de altura da planta na floração, as cultivares FHIA-21 e Thap Maeo apresentaram as maiores médias; no entanto, foram as que mais sofreram com os efeitos do vento. A altura é um descritor importante do ponto de vista fitotécnico e de melhoramento, pois influi nos aspectos de densidade de plantio e manejo da cultura, interferindo diretamente na produção. Embora a altura da planta só se estabilize no terceiro ano (ALVES, 2001), o primeiro ciclo já evidencia a tendência do comportamento da cultivar quanto a essa característica. A altura da planta também determina a maior ou menor facilidade na operação de colheita, podendo também influenciar no tombamento de plantas adultas. O tombamento de plantas e/ou quebra do pseudocaule pela ação do vento, além de estarem relacionados com o elevado porte, podem estar associados ao reduzido perímetro do pseudocaule e produção de cachos grandes.

$\mathrm{Na}$ Tabela 5, estão os dados do terceiro ciclo produtivo. Os valores de peso de cacho mostram que não houve incremento em relação ao segundo ciclo produtivo. No terceiro ciclo, a FHIA-1 e falsa FHIA-18 superaram as demais em termos de produção, como no segundo ciclo. Para o número de pencas, a Thap Maeo destacouse como nos ciclos anteriores. Quanto ao comprimento do engaço, destacaram-se a FHIA-1, falsa FHIA-18 e FHIA-21, como no segundo ciclo. No terceiro ciclo, com o bananal completamente estabelecido, foi possível observar redução do número de folhas vivas no florescimento em relação ao primeiro ciclo, em todas as cultivares, refletindo em produções semelhantes ao segundo ciclo. Com os dados dos três ciclos de avaliações, ficou bem característico o comportamento de cada genótipo nas condições locais. Todas as cultivares foram comercializadas na região, tendo a falsa FHIA-18, FHIA-21 e Thap Maeo a maior aceitação pelo mercado consumidor.

\section{CONCLUSÕES}

1) Os resultados obtidos neste trabalho qualificam as cultivares avaliadas como promissoras para serem incorporadas aos sistemas de produção do agricultor da região.

2) As condições do sudoeste de Goiás são favoráveis para a expressão da maioria dos caracteres avaliados, evidenciando adequação ambiental e/ou de manejo.

3) Para as cultivares FHIA-21 e Red Yade, recomendam-se monitoramento e controle da broca-da-bananeira pela alta suscetibilidade.

\section{AGRADECIMENTOS}

À Superintendência Federal de Agricultura de Goiás, AGÊNCIA RURAL e ao CNPMF-EMBRAPA, pelo apoio durante todo o projeto.

\section{REFERÊNCIAS}

ALVES, E. J. Cultivo da bananeira Tipo Terra. Cruz das Almas: EMBRAPA-CNPMF, 2001. 176p.

ANDRADE, G. M.; VASCONCELOS, L. F. L.; VELOSO, M. E. C.; SOUZA, V. A. B.; SOUSA, V. F. Avaliação de Genótipos de
Bananeira no Estado do Piauí. 1. Comportamento Vegetativo. In: CONGRESSO BRASILEIRODE FRUTICULTURA, 17., 2001, Belém. Anais... Belém: SBF, 2002.

DONATO, S. L. R.; SILVA, S. de O.; LUCCAFILHO, O. A.; LIMA, M. B.; DOMINGUES, H.; ALVES, J. da S. Comportamento de híbridos e variedades de bananeira (Musa spp.), em dois ciclos de produção no Sudoeste da Bahia. Revista Brasileira de Fruticultura, Jaboticabal, v.28, n.1, p.139-144, 2006.

FAO. FOOD AND AGRICULTURE ORGANIZATION OF THE UNITED NATIONS. THE WORLD BANANA ECONOMY 1985-2002, ROME, 2003. DISPONIVEL EM: <HTTP:// WWW.FAO.ORG/DOCREP/007/Y5102E/Y5102E00.HTM>. ACESSO EM: 20 ABRIL 2006.

HINZ, R. H. Moko e Sigatoka Negra. In: Curso sobre doenças da bananeira. Jataí - GO: DFA/GO, 2000. 9p.

MARIANO, Z. de F.; SCOPEL, I. Períodos de deficiências e excedentes hídricos na região de Jataí-GO. In: CONGRESSO BRASILEIRODEAGROMETEOROLOGIA, 12., 2001, Fortaleza. Anais... Fortaleza: SBA, 2001. p.333-34.

MOURA, R. J. M.; SILVA JÚNIOR, J. F.; SANTOS, V. F.; SILVA, S. O.; SÁ, V. A. L.; ANDRADE, O. J. L. Avaliação de Cultivares e Híbridos de Bananeira na Zona da Mata Norte de Pernambuco $\left(1{ }^{\circ} \mathrm{Ciclo}\right)$. In: CONGRESSO BRASILEIRODE FRUTICULTURA, 17., 2001, Belém. Anais... Belém: SBF, 2002.

OROZCO-SANTOS, M. Manejo integrado de la Sigatoka Negra del Plátano. Tecomán, Colima, México: SAGAR/INIFAP/CIPAC, 1998. 95p. (Folheto Técnico, 1)

PEREIRA, M. C. T.; SALOMÃO, L. C. C.; SILVA, S. O.; CECON, P. R.; PUSCHMANN, R.; JESUS, O. N.; CERQUEIRA, R. C. Suscetibilidade à queda natural e caracterização dos frutos de diversos genótipos de bananeiras. Revista Brasileira de Fruticultura, Jaboticabal, v.26, n.3, p.499-502, 2004.

RIBEIRO, A. C.; GUIMARÃES, P. T. G.; ALVAREZ V., V. H. (Ed.). Recomendação para o uso de corretivos e fertilizantes em Minas Gerais: 5. Aproximação. Viçosa: Comissão de Fertilidade do Solo do Estado de Minas Gerais, 1999. 359p.

ROSA, G. R; RIGON, L.; BELING, R. R.; CORRÊA, S.; REETZ, E.; VENCATO, A. Anuário Brasileiro de Fruticultura. Santa Cruz do Sul: Editora Gazeta, 2006. 136p.

SILVA, E. A.; BOLIANI, A.C.; CORRÊA, L. de S. Avaliação de cultivares de bananeira (Musa sp) na região de Selvíria-MS. Revista Brasileira de Fruticultura, Jaboticabal, v.28, n.1, p.101103, 2006.

SILVA, S. O.; FLORES, J. C.; LIMA NETO, F.P. Avaliação de cultivares e híbridos de bananeira em quatro ciclos de produção. Pesquisa Agropecuária Brasileira, Brasília, v.37, n.11, p.1.567-1.574, 2002.

SILVA, S. O.; ROCHA, S. A.; ALVES, E. J.; CREDICO, M.D.; PASSOS, A. R. Caracterização morfológica e avaliação de cultivares e híbridos de bananeira. Revista Brasileira de Fruticultura, Jaboticabal, v.22, n.2, 2000.

SILVA, S. de O.; SHEPHERD, K.; ALVES, E. J.; DANTAS, J. L. L. Cultivares de banana. In: ALVES, E. J. A cultura da banana: aspectos técnicos, socioeconômicos e agroindustriais. Brasília: EMBRAPA-SPI, 1999. p.85-105.

SEAGRO. SECRETARIA ESTADUAL DE AGRICULTURA, PECUÁRIA E ABASTECIMENTO DO ESTADO DE GOIÁS. Banana: Municípios Maiores Produtores em 2000, 2001 e 2002. Disponível em: http://www.agronegocio.goias.gov.br/docs/ portal/bananae.pdf. > . Acesso em: 23 março 2006. 